\title{
Improvement of Machinery Contact-type Sensor
}

\author{
Wenming Zhang, Jian Xu \\ Department of Mechanical Engineering, Shenyang University, Shenyang, 110044, China
}

\begin{abstract}
Keywords: Machinery Contract-type sensor; Regulating-wheel; Capacitive Sensor; Real-time Tracking
\end{abstract}

\begin{abstract}
This paper introduces an improving method to machinery contact-type sensor with pintype probe and capacitive sensor. The concrete improving process includes the content as follows: First, the V-type regulating wheel replaces the pin-type probe; second, the capacitive sensor replaces the displacement sensor. After improving, the capacitive sensor would burden the task of transformation which acted as the conversion device between welding seam offset and electrical signal offset, and the V-type probe would burden the task of testing the location of welding seam. This improving method would improve the availability of machinery contact-type sensor at the aspects of the linearity and the sensitivity as soon as possible. Consequently, the new-type machinery contact-type sensor can adapt the different tracking conditions with the state of real-time better under the complex and various environments.
\end{abstract}

\section{Introduction}

Narrow gap welding technology raised America Battelle institution in 1960s. However, after the efforts of many researchers, this technology underwent a high-speed development period and gradually walked to the state of mature at 1970s and 1980s [4]. Now this technology is stepping into the automatic times. Hence, a number of narrow gap seam automatic tracking systems appeared time by time, and some even have been put into the practice in the field of welding. The laser-based vision sensor seam tracking system has the widest usage range, because of the advantage of high tracking-precision [5]. What is more, the characteristic of real-time control to the welding conditions make this sensor can adapt all kinds of environments. However, influenced by smoke, arc light and bias light easily becomes the major disadvantage and limits its usage range. Besides, the complex principle and high cost of this sensor also make it not easy to mend when falls [1].

However, the machinery contact-type sensor would make up the disadvantage of easily influencing by environment with the better real-time tracking ability. The principle of acquiring seam deviation by machinery contact-type sensor includes the contents as follows: First, the pintype machinery probe attaches tightly the side wall of narrow gap seam because of the spring that connects pendulum with adjusting nut; second, when the deviation occurs, the angle of the pendulum changes, while the pin-type probe still attaches the side wall of narrow gap seam; third, the seam location deviation translates electricity signal by displacement sensor finally [2]. The outstanding advantage of this sensor is that it can avoid the welding atmosphere better as provided in former. However, this sensor also exposes some shortages in the practice, especially in the field. This paper introduces the improving plan to improve the machinery contact-type sensor and let it adapt the condition of field.

\section{Analysis of Machinery Contact-type Sensor}

The machinery contact-type sensor is shown in Figure 1. 


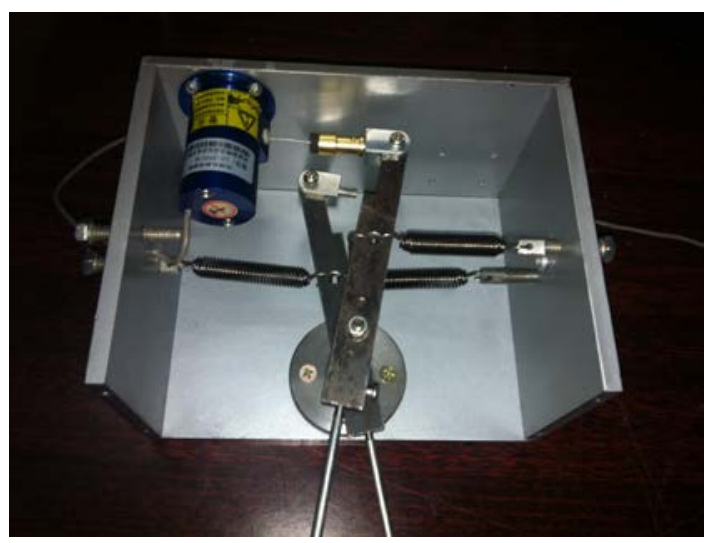

Figure 1 Machinery Contact-type Sensor

The working principle is shown in Figure 2.

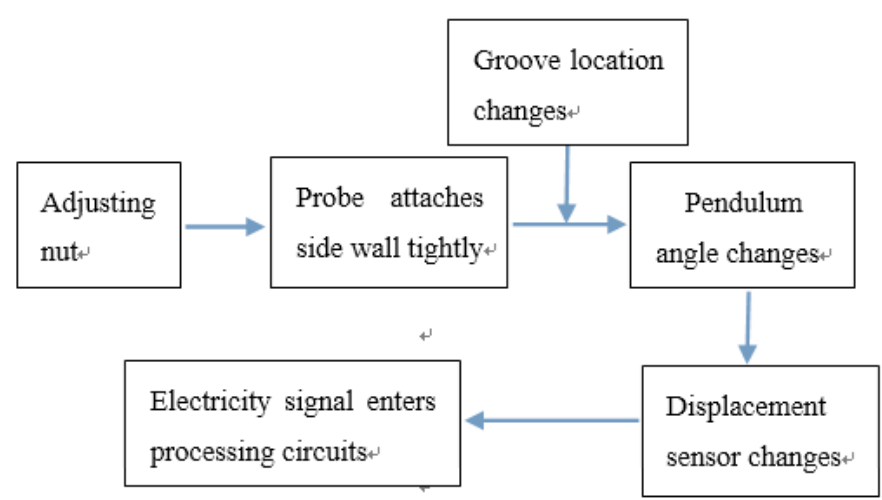

Figure 2 Machinery Contact-type Sensor working principle diagram

Figure 1 shows the wholly structures of machinery contact-type sensor. From it we can conclude that machinery contact-type sensor has three parts: adjusting device, machinery test device and signal conversion device. The adjusting device includes adjusting nut and spring, the machinery test device includes pendulum and pin-type probe. The pin-type probe is the first part of needing to improve. During tracking, if the processing precision of the seam groove is higher, the tracking precision is better; if the processing precision lower, the tracking precision is worse. Because the un-smooth of groove would make the probe tremble acutely, what is worse, the pin-type probe probably away from the side wall and fails to track finally. Therefore, the pin-type probe needs to improve with high-stable probe. In practice, especially in the field, the measure usually is that the pin-type probe would be replaced by the $\mathrm{V}$-type regulating wheel probe with higher flexibility.

The second part of needing to improve is the signal conversion device. Comparing Figure 1 and Figure 2 we know that the signal conversion device is the terminal of the sensor and the bond of sensor and signal control system, so the performance of the signal conversion device has the critical influence to the quality of acquiring signal. Figure 2 shows that the displacement sensor as the signal conversion device in the machinery contact-type sensor, its function is to translate the signal between seam location deviation and electricity signal deviation. The displacement sensor includes four parts: spring, bobbin winder, potentiometer and movable end. The working principle attains the contents as follows: First, the length of movable end changes by pendulum; second, the bobbin winder rotates by the movable end; third, the resistance of potentiometer changes and the voltage value also changes. After these series of processes, the seam location deviation translates into the voltage signal. Although displacement and resistance are linear in the condition of simplifying, in field, the relation is very complex, and it would be influenced easily by the all kinds of environment factors. Therefore, the sensitivity and precision are lower than expected, especially the sensitivity. A better sensor is acquired to the tracking system. During optimization, the displacement sensor is replaced by capacitive sensor by the better performance. 


\section{Regulating Wheel Probe}

During the improving method, the pin-type probe is replaced by the regulating wheel probe. The regulating wheel uses the softer $V$-type rubber-wheel to attach the side wall of narrow gap seam, to strength the effort, a thin layer of sponge packages outside of the regulating wheel. The intention contains the contents as follows: First, the softer rubber wheel would form by the pressure and avoid changing the wheel frequently according the shape of seam groove; second, the thin layer of sponge would protect the rubber wheel, in case of melting because of high temperature. However, the fundamental role is stable, it is the probe that attaches the side wall of narrow gap seam tightly by the spring and measures the seam deviation by the rolling of pendulum. The difference is lower processing precision to the seam groove, at the same time, the cost is lower. But this V-type rubber regulating wheel probe still has some shortages, such as the rubber does not support higher temperature; it is out of shape easily, etc. These would be dealt in the post-processing.

\section{Capacitive Sensors}

\section{Feasibility Analysis of Capacitive sensors}

The fundamental formula of capacity acquired by physics is [3]:

The incremental formula of capacity acquired by (1) is:

$$
C=\frac{\varepsilon S}{\delta}=\frac{\varepsilon_{1} \varepsilon_{2} S}{\delta}
$$

$$
C_{0}+\Delta C=\frac{\varepsilon_{1} \varepsilon_{2} S}{\delta_{0}-\Delta \delta_{0}}=\frac{C_{0}}{1-\Delta \delta_{0} / \delta_{0}}
$$

(2) Shows that the output characteristic of capacity approaches the hyperbola rather than linear. Consequently, the capacity increment does not act as the output characteristic.

Combining (1) and (2) can get the relative increment formula of capacity is:

$$
\Delta C / C_{0}=\frac{\Delta \delta_{0}}{\delta_{0}}\left(1-\frac{\Delta \delta_{0}}{\delta_{0}}\right)^{-1}
$$

Because of $\Delta \delta_{0} / \delta_{0} \ll 1$, the (3) can simplify further is:

$$
\Delta C / C_{0} \approx \frac{\Delta \delta_{0}}{\delta_{0}}
$$

(4) Shows that the gap changes between two capacity plates can be expressed by the changes of capacity value, so the plan which capacity sensor replaces displacement sensor is feasible.

\section{Capacitive Sensor Model}

Plate material, plate gap, plate thickness and the edge effect of parallel plate capacity would influence the capacity sensor easily. These parameters must be sure by data book, and the more precise parameters would through a series of experience.

The selection principle of capacity plate material comes two aspects. First, considering sensitivity and precision, the initial capacity value should as large as possible. Second, considering the material itself, the ability of thermal expansion and contracting of material should as small as possible. The data is shown in Table 1. Comparing different experience data, the fixed plate chooses the steel plate; the moving plate is copper clad laminate.

Table 1: Capacity value of different materials

\begin{tabular}{|l|l|l|l|l|}
\hline Material/Location & 20 & 30 & 40 & 50 \\
\hline $\mathrm{Cu}-\mathrm{Al}$ & 6.0 & 3.5 & 3.1 & 2.0 \\
\hline $\mathrm{Cu}-\mathrm{Iron}$ & 20.0 & 12.5 & 9.0 & 6.1 \\
\hline Iron-Al & 18.6 & 12.1 & 8.3 & 5.7 \\
\hline $\mathrm{Cu}-\mathrm{Cu}$ & 6.3 & 4.2 & 2.5 & 1.9 \\
\hline
\end{tabular}


(1) Shows that the smaller gap between capacity plates, the larger capacity value. However, if the gap too small, the capacity would be destroyed by the voltage breakdown. On the other hand, considering measuring processing, the suitable gap between plates is necessary. The data is shown in Table 2 . Therefore, the gap chooses $0.8 \mathrm{~mm}$ finally by considering all factors and checking data book.

Table 2: Capacity value of different gaps

\begin{tabular}{|l|l|l|l|l|}
\hline Gap/Location & 20 & 30 & 40 & 50 \\
\hline $0.5 \mathrm{~mm}$ & 14.7 & 10.1 & 7.5 & 5.2 \\
\hline $1.0 \mathrm{~mm}$ & 7.0 & 4.7 & 3.5 & 2.1 \\
\hline $2.0 \mathrm{~mm}$ & 3.9 & 2.3 & 0.8 & 0.1 \\
\hline
\end{tabular}

The influencing factors of capacity plate thickness include two aspects: initial capacity and parasitic capacitance. These two factors all would influence the non-linear error of capacity sensor. The data is shown in Figure 3. Considering these factors and referencing the data from data book, capacity plate thickness chooses $0.7 \mathrm{~mm}$.

Table 3: Capacity value of different thicknesses

\begin{tabular}{|l|l|l|l|l|}
\hline Thickness/Location & 20 & 30 & 40 & 50 \\
\hline $0.2 \mathrm{~mm}$ & 11.8 & 9.6 & 6.5 & 4.8 \\
\hline $0.5 \mathrm{~mm}$ & 18.5 & 12.1 & 7.9 & 5.1 \\
\hline $1.0 \mathrm{~mm}$ & 7.5 & 4.9 & 3.7 & 2.8 \\
\hline
\end{tabular}

The edge effect of parallel plate capacity would let the non-linear error aggravate, because the electric field in the edge of capacity would become more uneven. For reducing or eliminating this influence, the measure of adding shielding layer in the edge of parallel plate capacity is added.

\section{Differential Measurement Method}

The differential measurement method, acted as the method of improving the test precision and reducing non-linear error, the principle is that the balance keeps when the moving plate in the medium between two fixed plates, the balance fails once the moving plate moves.

When the moving plate locates the point of balance, the distance between moving plate and fixed plates all are ${ }^{\delta_{0}}$; when it towards left $\Delta \delta_{0}$, the distance between moving plate and left fixed plate is $\delta_{0}-\Delta \delta_{0}$, the distance between moving plate and right fixed plate is $\delta_{0}+\Delta \delta_{0}$, so the non-linear error of capacitive sensor acquired is:

$$
\mathrm{e}_{\mathrm{f}}=\left|\frac{\Delta \delta_{0}}{\delta_{0}}\right|^{2} \times 100 \%
$$

However, the non-linear error which the differential measurement method is not used is:

$$
\mathrm{e}_{\mathrm{f}}=\left|\frac{\Delta \delta_{0}}{\delta_{0}}\right| \times 100 \%
$$

By comparing (5) and (6), it shows that the non-linear error is lower and the sensitivity is higher to capacitive sensor when the differential measurement is used.

\section{Conclusions}

This paper intends to make the machinery contact-type sensor has the higher linearity and sensitivity by optimizing mechanical probe with V-type regulating wheel and replacing the displacement sensor with capacity sensor, and then further to improve the sampling precision of deviation signal. Confirmed by experience, the result agrees with the supposed result better we have expected, what is more, the ability of anti-interference has strengthened further, so it can adapt all kinds of environment better.

However, the improved machinery contact-type sensor still has some shortages, such as for the 
regular seam groove, the tracking effect is better, for the un-regular, the effect is still worse; the non-linear error still cannot be neglected completely; the edge effect still has the obvious influence; the rubber regulating wheel is easy to melt etc. These shortages still need to improve further and the more improved sensor would enter into the tracking system.

\section{Acknowledgements}

This research work was supposed energetically by the first author-Wen-ming Zhang professor. Under the indication of Professor Zhang, we overcame many difficulties and obstacles that we are not expected, what is more, Professor Zhang infuse much new knowledge to us during this process. At the same time, we also express the thanks to the people of providing all kinds of knowledge to this search work.

\section{References}

[1] Bo Hong, Junguang Yan, Jiawang Yang, Xiang Liu. A Capacitive Sensor for Seam-tracking [J]. Transactions of The China Welding Institution, 2014, 35 (2): 55-58.

[2] Bin Wang, Jiaqi Yang. Design on Contact Type Seam Tracking Sensor in Narrow Gap Welding [J]. Hot Working Technology, 2014, 43 (15): 208-209.

[3] Qianhong Wang. Principle and Measuring Circuits of Capacitive Sensor [J]. Science and Technology Monograph: 192.

[4] Meister R P, Martin D C. Narrow-gap Welding Process [J]. British Welding Journal, 1966, 13 (5): 252-257.

[5] Fuju Zhang, Chuanhong Luo. Narrow-gap Welding and New Processes [J]. Welding Technology, 2000, (6): 33-35. 